\title{
Culture-Independent Genotyping Revealed 3 Strain Clusters in a Potential Neisseria gonorrhoeae Outbreak in Young Heterosexuals (<25 Years), the Netherlands, October 2017 to March 2019
}

Citation for published version (APA):

van der Veer, B. M. J. W., Wolffs, P. F. G., Hoebe, C. J. P. A., Wijers, J. N. A. P., van Liere, G. A. F. S., Werner, M. I. L. S., Verhaegh, A., Dukers-Muijrers, N. H. T. M., \& van Alphen, L. B. (2021). CultureIndependent Genotyping Revealed 3 Strain Clusters in a Potential Neisseria gonorrhoeae Outbreak in Young Heterosexuals ( $<25$ Years), the Netherlands, October 2017 to March 2019. Sexually Transmitted Diseases, 48(8), 536-541. https://doi.org/10.1097/olq.0000000000001370

Document status and date:

Published: 01/08/2021

DOI:

10.1097/olq.0000000000001370

Document Version:

Publisher's PDF, also known as Version of record

Document license:

Taverne

Please check the document version of this publication:

- A submitted manuscript is the version of the article upon submission and before peer-review. There can be important differences between the submitted version and the official published version of record. People interested in the research are advised to contact the author for the final version of the publication, or visit the DOI to the publisher's website.

- The final author version and the galley proof are versions of the publication after peer review.

- The final published version features the final layout of the paper including the volume, issue and page numbers.

Link to publication

\footnotetext{
General rights rights.

- You may freely distribute the URL identifying the publication in the public portal. please follow below link for the End User Agreement:

www.umlib.nl/taverne-license

Take down policy

If you believe that this document breaches copyright please contact us at:

repository@maastrichtuniversity.nl

providing details and we will investigate your claim.
}

Copyright and moral rights for the publications made accessible in the public portal are retained by the authors and/or other copyright owners and it is a condition of accessing publications that users recognise and abide by the legal requirements associated with these

- Users may download and print one copy of any publication from the public portal for the purpose of private study or research.

- You may not further distribute the material or use it for any profit-making activity or commercial gain

If the publication is distributed under the terms of Article 25fa of the Dutch Copyright Act, indicated by the "Taverne" license above,

Download date: 26 Apr. 2023 


\title{
Culture-Independent Genotyping Revealed 3 Strain Clusters in a Potential Neisseria gonorrhoeae Outbreak in Young Heterosexuals ( $<25$ Years), the Netherlands, October 2017 to March 2019
}

\author{
Brian M.J.W. van der Veer, MSc, * Petra F.G. Wolffs, PhD, * Christian J.P.A. Hoebe, MD, PhD, *† \\ Juliën N.A.P. Wijers, MSc, *† Gènevieve A.F.S. van Liere, PhD, *† Marita I.L.S. Werner, MD, † \\ Amanja Verhaegh, MD, $\dagger$ Nicole H.T.M. Dukers-Muijrers, PhD, $*$ $\neq$ and Lieke B. van Alphen, PhD*
}

\begin{abstract}
Background: Investigation was undertaken to determine the genetic relatedness of Neisseria gonorrhoeae $(\mathrm{NG})$ isolates of young $(<25$ years $)$ heterosexuals of a potential outbreak from October 2017 to March 2019 in South-Limburg, the Netherlands.

Methods: Data and residual sample material of routine diagnostics were retrieved for outbreak cases (78/81), young heterosexuals at baseline (January 2016 to September 2017, $\mathrm{n}=30$ ), and men who have sex with men (2018, $\mathrm{n}=47$ ). Total DNA was isolated, and NG was genotyped using culture-free NG multiantigen sequence typing. Sanger sequence data were used to construct a phylogenetic tree. Cases of outbreak clusters were geographically mapped, and descriptive analyses were performed on patient characteristics, comparing these clusters.

Results: Outbreak investigation showed 81 cases of young heterosexuals between October 2017 and March 2019 (4.5 per month) compared with 30 between January 2016 and September 2017 (1.4 per month), which was considered as baseline. Culture-independent genotyping of NG was performed to assess the genetic relatedness, as only 21 outbreak cases were culture confirmed. This revealed 3 independent outbreak clusters G2 $(\mathrm{n}=18)$, G13113 ( $n=11)$, and GNewST $(n=24)$. None of the clusters were geographically linked or introduced by bridging with men who have sex with men networks. Number of sex partners reported by men and Chlamydia trachomatis coinfection were associated with clusters G2 and GNewST, respectively. Conclusions: Culture-independent typing proved to be essential to identify the 3 outbreak clusters. However, targeted interventions were difficult because information on sex partners was limited. Therefore, prospective cultureindependent typing could be used for early outbreak detection and aid in transmission prevention.
\end{abstract}

$N$ eisseria gonorrhoeae (NG) is a common bacterial sexually transmitted infection (STI) that can infect genital, anorectal,

From the *Department of Medical Microbiology, Care and Public Health Research Institute (CAPHRI), Maastricht University Medical Centre (MUMC+), Maastricht; $\dagger$ Department of Sexual Health, Infectious Diseases and Environmental Health, South Limburg Public Health Service, Heerlen; and \$Department of Health Promotion, Care and Public Health Research Institute (CAPHRI), Maastricht University Medical Centre (MUMC+), Maastricht, the Netherlands

Conflict of Interest and Sources of Funding: None declared.

Correspondence: Lieke B. van Alphen, PhD, Maastricht University Medical Centre (MUMC+), PO Box 5800, 6202 AZ Maastricht. E-mail: lieke.van.alphen@mumc.nl.

Received for publication October 21, 2020, and accepted December $15,2020$.

Supplemental digital content is available for this article. Direct URL citations appear in the printed text, and links to the digital files are provided in the HTML text of this article on the journal's Web site (http://www.stdjournal.com). DOI: 10.1097/OLQ.0000000000001370

Copyright (C) 2021 American Sexually Transmitted Diseases Association. All rights reserved. oropharyngeal, and ocular mucosa. ${ }^{1-3}$ Many NG infections, especially anorectal and oropharyngeal infections, are asymptomatic, which could lead to unnoticed transmission. ${ }^{4,5}$ Therefore, it is a major public health challenge as the incidence rate remains high despite improved diagnostics. ${ }^{1,6}$ Key populations with a higher risk of acquiring NG such as men who have sex with men (MSM) have been identified. ${ }^{4}$ This is reflected in a higher positivity rate among MSM (11.0\%) compared with women (1.6\%) and heterosexual men $(1.8 \%)$ in the Netherlands. ${ }^{7}$

Molecular epidemiology can have an important contribution to the elucidation of sexual networks, in addition to collected epidemiological data. ${ }^{8} \mathrm{~N}$. gonorrhoeae multiantigen sequence typing (NG-MAST) and whole-genome sequencing have been applied to elucidate transmission networks and outbreaks of NG. ${ }^{9-16}$ These studies revealed sustained transmission of some lineages in both MSM and heterosexuals resulting in strain clusters. ${ }^{9,10,12-14,16}$ Also, bridging between MSM and heterosexual sexual networks has been demonstrated previously. ${ }^{13}$ Furthermore, some studies reveal outbreaks of NG in distinct sexual networks or geographical areas. ${ }^{9,10,15}$ However, the previous studies relied on culture of NG that frequently fails. ${ }^{9-16}$ Therefore, some links between individuals could be missed if no cultured isolates are available.

We noticed a potential outbreak of NG in the young $(<25$ years $)$ heterosexual population visiting our STI clinic. We observed an increasing number of young women with genital NG and an increased number of young heterosexual men, whereas triage and the number of tests did not change since $2015 .{ }^{17}$ In the last quarter of 2017, 7 young women were diagnosed with genital NG visiting our STI clinic, whereas in the previous three-quarters of 2017, only 5 young women were diagnosed with genital NG. Here, we report the main findings of molecular outbreak investigation and demonstrate the added value of culture-independent genotyping and discuss challenges associated with NG outbreak detection and management.

\section{METHODS}

\section{Case Definition and Data Collection}

Confirmed cases related to this outbreak were defined as young $(<25$ years) heterosexuals (women and men) living in the region South-Limburg in the Netherlands with a genital NG infection from October 2017 to March 2019. Extragenital samples were not included because heterosexuals are not routinely tested at those anatomical sites as recommended by the Dutch STI guidelines. ${ }^{17}$ N. gonorrhoeae and Chlamydia trachomatis (CT) infections were diagnosed in routine diagnostics using the Cobas 4800 CT/NG test (Roche Diagnostics, Basel, Switzerland) using a self-collected vaginal swab (women) or first-void urine sample 
(men). As part of routine diagnostic procedures, a standardized questionnaire regarding the medical and sexual history of the last 6 months was collected by trained nurses. Data on sexual risk group, symptoms, number of sex partners, educational level, residence (4-digit postcode), and ethnicity were used in this study. The following were considered as urogenital symptoms: genital discharge, bleeding, itching, swelling, pain, burning sensation, and more frequent urination. Educational level was defined as low/ medium (no education, primary school, or vocational) and high (higher education [secondary school] or university). Ethnicity was categorized as Western and non-Western background according to the Dutch consultation manual. ${ }^{17}$ All missing data were handled as unknown and were not included in the analyses.

\section{Outbreak Investigation}

There were 81 confirmed cases ( 57 women and 24 men) in the time frame of October 2017 to March 2019 compared with 30 young heterosexuals (17 women and 13 men) with genital NG from January 2016 to September 2017, which were considered as baseline. This resulted in a 3-fold higher number of genital NG-positive young heterosexuals per month during the outbreak (4.5 per month) compared with baseline (1.4 per month). Three outbreak cases were excluded because of the lack of residual material for genotyping, leaving 78 cases ( 56 women and 22 men), of which $21(26.9 \%)$ were culture confirmed, available for analysis. Residual samples of routine diagnostics, stored at $-20^{\circ} \mathrm{C}$ till use (within 1 year), were genotyped using culture-free NG-MAST to assess the genetic relatedness of the NG strains. ${ }^{18}$ For comparison, residual NG-positive genital material of young heterosexuals at baseline was genotyped to determine the occurrence of clustering in this population. In addition, residual NG-positive genital material of MSM, including bisexual men, in $2018(n=47)$ was genotyped to check for potential introduction of NG in the heterosexual population via bridging.

\section{Culture-Independent Genotyping}

DNA isolation and culture-free NG-MAST were performed as previously described, with minor modifications to the polymerase chain reaction (PCR) protocol. ${ }^{18}$ Polymerase chain reaction protocols were modified as follows to improve sensitivity. The por $B$ PCR protocol: 5 minutes at $95^{\circ} \mathrm{C}, 12$ cycles of 30 seconds at $95^{\circ} \mathrm{C}, 3$ minutes at $68^{\circ} \mathrm{C}$ (decreasing by $0.5^{\circ} \mathrm{C}$ per cycle), $30 \mathrm{cy}$ cles of 30 seconds at $95^{\circ} \mathrm{C}, 1$ minute at $58^{\circ} \mathrm{C}, 2.5$ minutes at $68^{\circ} \mathrm{C}$, and a final extension of 10 minutes at $68^{\circ} \mathrm{C}$. The $t b p B$ PCR protocol: 5 minutes at $95^{\circ} \mathrm{C}, 12$ cycles of 30 seconds at $95^{\circ} \mathrm{C}, 3$ minutes at $72^{\circ} \mathrm{C}$ (decreasing by $0.5^{\circ} \mathrm{C}$ per cycle), 30 cycles of 30 seconds at $95^{\circ} \mathrm{C}, 1$ minute at $65^{\circ} \mathrm{C}, 2.5$ minutes at $68^{\circ} \mathrm{C}$, and a final extension of 10 minutes at $68^{\circ} \mathrm{C}$.

\section{Data Analysis}

The Sanger sequence data analysis was performed as described previously. ${ }^{18}$ Bionumerics version 7.6 (Applied Maths, Sint-Martens-Latem, Belgium) was used to perform multiple sequence alignment and unweighted pair group method with arithmetic mean clustering on concatenated $\operatorname{por} B$ and $t b p B$ sequences as described previously. ${ }^{18}$ Genogroups were assigned if $\geq 10$ cases had a shared identical allele and the other $\geq 99 \%$ similarity and were considered as a cluster. ${ }^{19}$ Genogroups were used to ensure robust statistical analysis as proposed by Chisholm et al. and because some known sexual contacts do not have identical NG-MAST sequence types. $^{19,20}$

Geospatial coordinates (residence) of outbreak cases were extracted using the R-package "nlgeocoder" version 0.1.3. We visualized residence areas of patients, based on their 4-digit postcode, per outbreak cluster in a geographical map using Qgis 2.18.28. ${ }^{21}$ In addition, the coordinates were used to test whether outbreak clusters, based on NG-MAST, were associated with a geographical area using SatScan (version 9.6) using the multinomial model. ${ }^{22}$
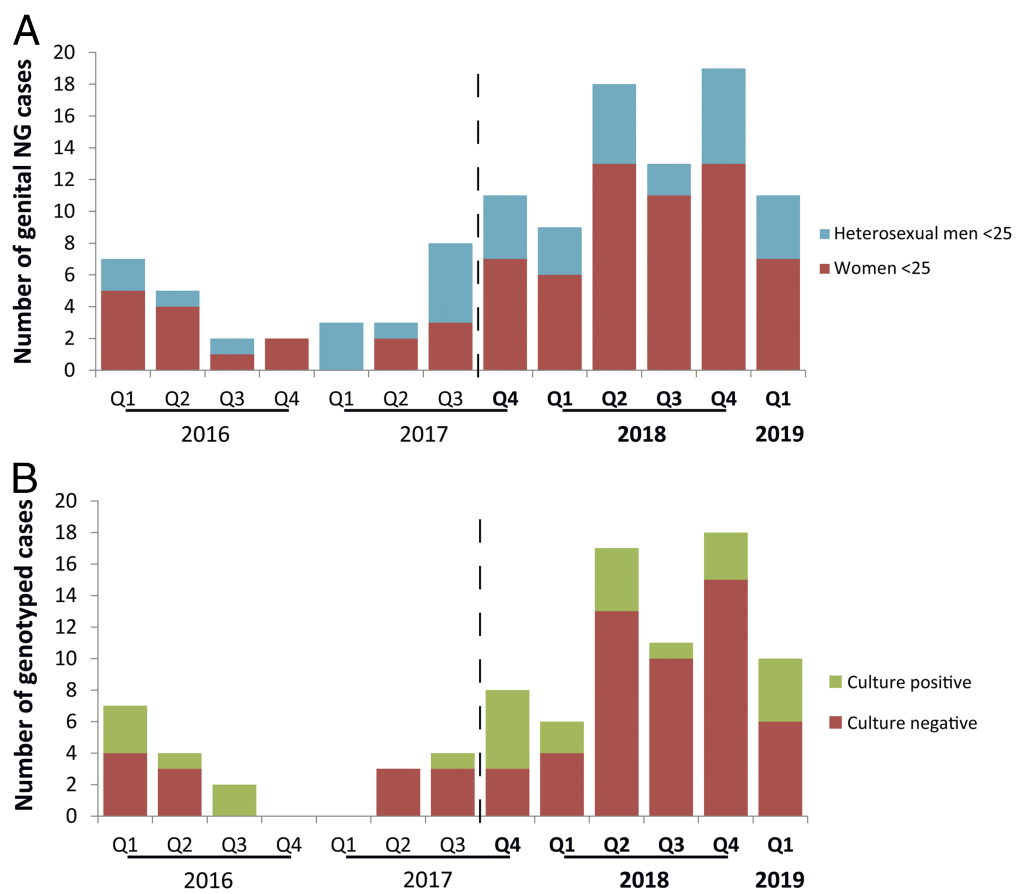

Figure 1. A, Epidemiological curve of genital NG cases in young heterosexuals in South-Limburg, the Netherlands. B, Epidemiological curve of genotyped samples of young heterosexuals in South-Limburg, the Netherlands. Bars are colored according to the availability of cultured isolate. The dashed vertical line and the bold dates indicate the outbreak. 
Differences in epidemiological characteristics (sex, CT coinfection, educational level, notified for an STI, symptoms, and ethnicity) between strain clusters were pairwise compared with the Fisher exact test. Student $t$ test was used to test associations with number of sex partners between strain clusters and between women and men within a strain cluster. These analyses were performed using $\mathrm{R}$ statistical software version 3.6.2 (R Foundation for Statistical Computing, Vienna, Austria).

\section{Ethical Statement}

The data in this study were obtained from medical records from routine care of our STI clinic in a deidentified manner. None of the researchers had access to patient-identifying information. The STI clinic uses the "no objection" informed consent, and persons who refused use of data or samples are not included in this study. The study protocol was exempt from formal medical-ethical approval under prevailing laws in the Netherlands, as it concerns only an retrospective observational study using anonymous data only (as stated by the National Central Committee for Human
Studies [www.ccmo.nl] and in the conduct of good behavior in research [www.federa.org]).

\section{RESULTS}

\section{Descriptive Epidemiology}

A total of 81 cases were identified ( 57 women and 24 men) in the time frame of October 2017 to March 2019 (Fig. 1A). As the increase in genital NG cases in young women was notified in the last quartile of 2017, all young heterosexuals with genital NG from January 2016 to September 2017 were considered baseline $(n=30$, 17 women and 13 men; Fig. 1A). Of the potential outbreak cases, 3 were excluded because of the lack of residual material for genotyping, but none of the baseline as sufficient residual material was available.

\section{Three Independent Outbreak Clusters}

In total, $128(82.6 \%)$ of 155 genital NG-positive samples were successfully genotyped with culture-free NG-MAST, 20

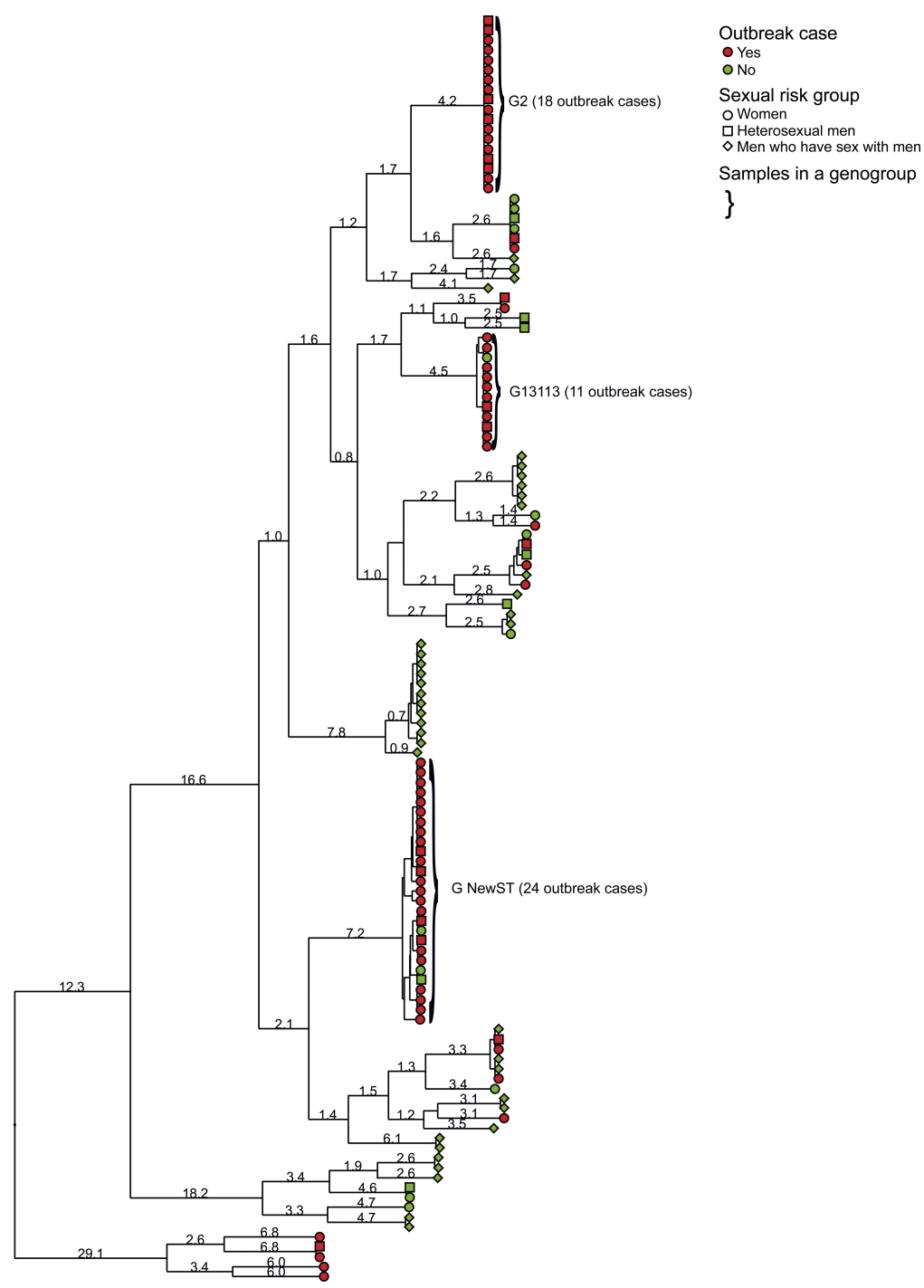

Figure 2. Dendogram of concatenated $p o r B$ and $t b p B$ sequences of outbreak (dark circles) and reference (white circles) cases, clustered with unweighted pair group method with arithmetic mean algorithm. Brackets indicate a genogroup and are named by the most abundant sequence type. The branch length depicts the percentage of dissimilarity. 


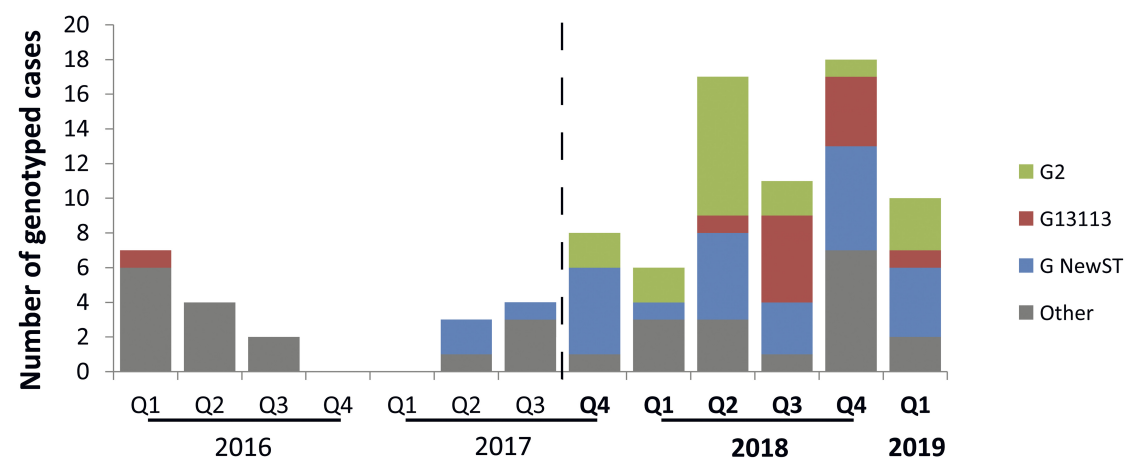

Figure 3. Epidemiological curve of genotyped samples of young heterosexuals in South-Limburg, the Netherlands. Bars are formatted according to genogroup. The dashed vertical line and the bold dates indicate the outbreak.

(66.7\%) of 30 samples at baseline, $70(89.7 \%)$ of 78 confirmed outbreak cases, and $38(80.9 \%)$ of 47 MSM. Of the genotyped samples, only $57(44.5 \%)$ of 128 were culture positive. Of the 27 samples that failed genotyping (8 vaginal swabs and 19 urine samples), 5 showed potential mixed strain infections (4 culturepositive) and 8 had a low bacterial $\operatorname{load}_{(<2.8} \log _{10} \mathrm{CFU} / \mathrm{mL}$, no culture-positives). The remaining 14 samples ( 2 culture-positive) could have failed because of PCR inhibition. Most genotyped samples at baseline and during outbreak were not culture confirmed and thus could only be typed by culture-independent methods (Fig. 1B). Figure 2 presents the dissimilarity of concatenated por $B$ and $t b p B$ sequences revealing 3 independent outbreak clusters, namely, G2 $(\mathrm{n}=18)$, G13113 $(\mathrm{n}=11)$, and GNewST $(\mathrm{n}=24)$. Of these outbreak clusters, only 5 of 18, 2 of 11, and 6 of 24 of cases had a cultured isolate and therefore would not have met the outbreak cluster criteria if a culture-dependent method was used. Genogroups, G13113 ( $\mathrm{n}=1)$ and GNewST $(\mathrm{n}=3)$, were already present in the young heterosexual population before the increase
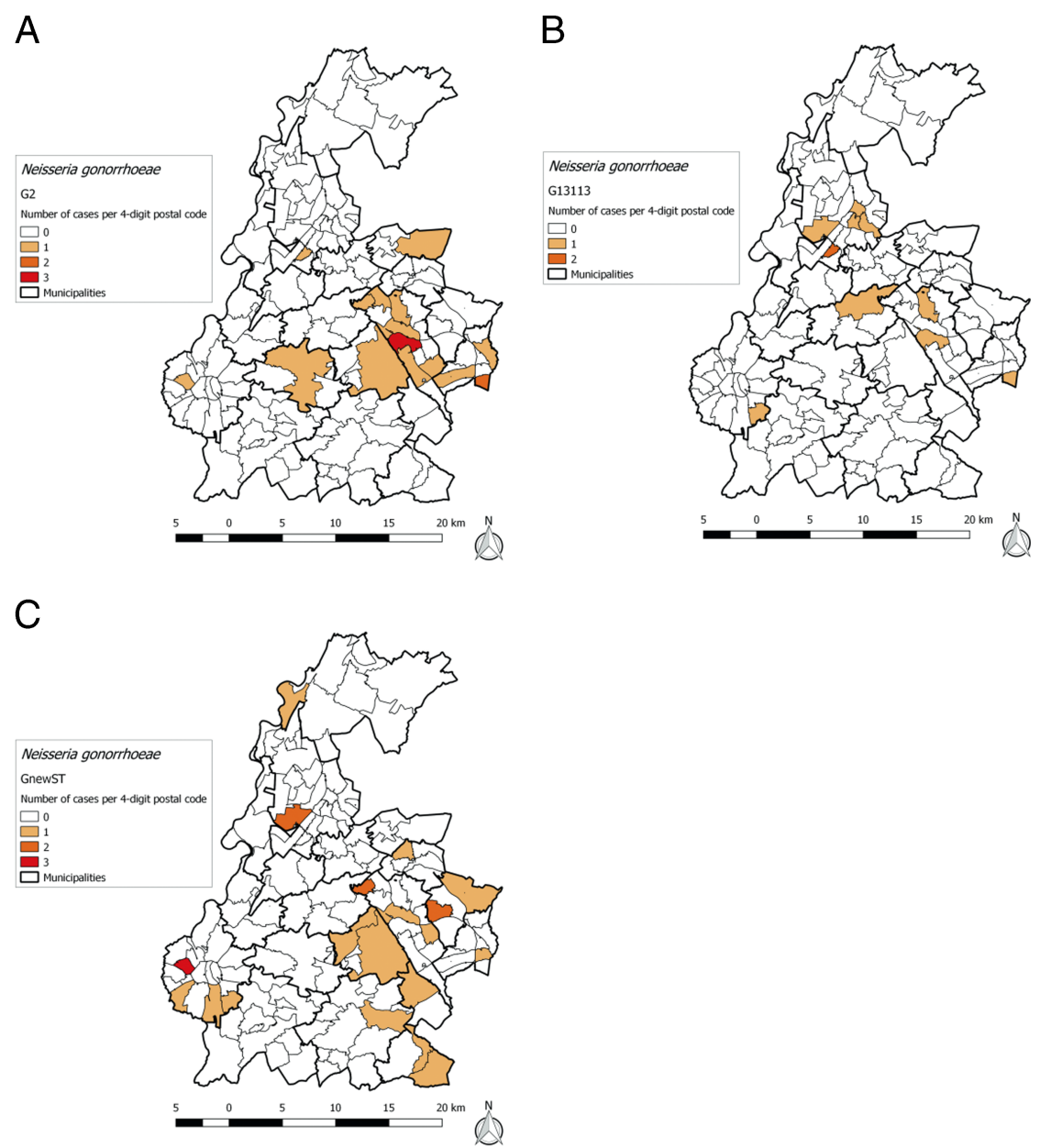

Figure 4. Geographical maps of cases per outbreak cluster. The black lines represent the municipalities, and the gray dots represent the geographic area of the 4-digit postal code. 
of genital NG, in January 2016 (G13113) and between May and September 2017 (GNewST), respectively (Fig. 3). No genogroups were seen when solely using preoutbreak young heterosexual cases and MSM cases (Fig. 2).

\section{Geographical Mapping}

The 4-digit postcode of patients per outbreak cluster was used to explore possibilities for targeted interventions. Figure 4 shows that the cases per outbreak cluster are quite scattered in South-Limburg. Despite the small numbers, geographical clustering analysis was performed using SatScan, but no significant geographical clustering was observed (Supplemental Table, http:// links.lww.com/OLQ/A612).

\section{Association of Epidemiological Characteristics With Outbreak Clusters}

Because no clear geographical clustering was observed, other explanatory variables could provide insight into the epidemiology of the outbreak. Therefore, we analyzed whether a significant association with sex, sex partner, CT coinfection, educational level, notification for an STI, symptoms, and ethnicity could be assessed. Fisher exact tests showed that CT coinfection was significantly associated with cluster GNewST $(92 \%, P<0.03$; Table 1$)$. Higher number of sex partners was reported by heterosexual men in cluster G2 compared with the other clusters $(P=0.03$, Table 1$)$. In addition, women in cluster G2 and G13113 report a lower number of sex partners compared with heterosexual men in the respective cluster $(P=0.03$ and $P=0.02)$.

\section{No Introduction of NG by Bridging}

The relatively high number of NG cases in the young heterosexual population could be the result of acquisition of NG by bridging with a high-risk population. Because MSM, including bisexual men, are a group at risk of acquiring an NG infection, bisexual men could introduce NG strains in the young heterosexual population. None of the cases of MSM, including bisexual men, clustered together with any of the 3 independent outbreak (Fig. 2). However, there were 3 smaller clusters of 6 individuals; 2 contained MSM, of which 1 also contained bisexual men (Fig. 2).

\section{Outbreak Control Measures}

Implementation of control measures to stop further transmission of NG within this population proved difficult because of limited information on sexual partners and no obvious association with a geographical area. A prospective approach was taken in which an additional questionnaire is implemented, which will be collected upon return to the clinic for treatment or via telephone when empirically treated. This questionnaire covers detailed information of all sex partners, sexual practices per sex partner, and where and when did they meet their sex partner(s). Furthermore, prospective culture-independent genotyping of NG-positive heterosexuals was implemented, as it proved essential for outbreak clusters identification. These prospectively collected data of the additional questionnaire and genotyping can be used to perform network analyses for targeted interventions to control future NG outbreaks.

\section{DISCUSSION}

Between October 2017 and March 2019, a 3-fold higher number of genital NG-positive young heterosexuals per month was seen (4.5 per month) compared with January 2016 to September 2017 (1.4 per month, considered as baseline), indicating a potential outbreak of NG among young heterosexuals, whereas NG is mostly diagnosed in MSM. Therefore, culture-independent genotyping was performed and revealed 3 independent outbreak clusters of only heterosexual individuals. Using culture-independent genotyping was essential to identify the 3 outbreak clusters, as only 5 of 18 (G2), 2 of 11 (G13113), and 6 of 24 (GNewST) cases had a cultured isolate available for typical culture-dependent genotyping. Analysis of these

TABLE 1. Characteristics of the Outbreak Clusters

\begin{tabular}{|c|c|c|c|}
\hline & G2 $(n=18)$ & G13113 $(n=11)$ & GNewST $(n=24)$ \\
\hline \multicolumn{4}{|l|}{ Sex } \\
\hline Women & $66.7(12)$ & $81.8(9)$ & $83.3(20)$ \\
\hline Men & $33.3(6)$ & $18.2(2)$ & $16.7(4)$ \\
\hline Sex partners reported by women & $2.3 \pm 1.2$ & $2.1 \pm 1.3$ & $2.6 \pm 2.1$ \\
\hline Sex partners reported by men & $11.3 \pm 7.3^{* \dagger}$ & $5.5 \pm 0.7^{*}$ & $2.8 \pm 2.4$ \\
\hline \multicolumn{4}{|l|}{ CT coinfection } \\
\hline CT negative & $38.9(7)$ & $54.5(6)$ & $8.3(2)$ \\
\hline CT positive & $61.1(11)$ & $45.5(5)$ & $91.7(22)^{*}$ \\
\hline \multicolumn{4}{|l|}{ Educational level } \\
\hline Low/medium & $66.7(12)$ & $90.9(10)$ & $66.7(16)$ \\
\hline High & $33.3(6)$ & $9.1(1)$ & $20.8(5)$ \\
\hline Unknown & $0(0)$ & $0(0)$ & $12.5(3)$ \\
\hline \multicolumn{4}{|l|}{ Notified for an STI } \\
\hline Not notified & $77.8(14)$ & $45.5(5)$ & $62.5(15)$ \\
\hline Notified & $22.2(4)$ & $45.5(5)$ & $37.5(9)$ \\
\hline Unknown & $0.0(0)$ & $9.1(1)$ & $0.0(0)$ \\
\hline \multicolumn{4}{|l|}{ Symptoms } \\
\hline Asymptomatic & $16.7(3)$ & $27.3(3)$ & $45.8(11)$ \\
\hline Symptomatic & $83.3(15)$ & $72.7(8)$ & $54.2(13)$ \\
\hline \multicolumn{4}{|l|}{ Ethnicity } \\
\hline Western & $88.9(16)$ & $81.8(9)$ & $95.8(23)$ \\
\hline Not-Western & $11.1(2)$ & $18.2(2)$ & $4.2(1)$ \\
\hline
\end{tabular}

Data are reported as percentages (number of cases) except sex partners, which are reported as mean (SD).

$* P<0.05$.

${ }^{\dagger}$ Men report more sex partners compared with women $(P=0.03)$.

${ }^{*}$ Men report more sex partners compared with women $(P=0.02)$. 
clusters showed an association of number of sex partners reported by men with cluster $\mathrm{G} 2$ and an association of CT coinfection with cluster GNewST. Based on our data set, the 3 independent strain clusters were not introduced by bridging with MSM sexual networks, as no MSM samples were identified in the outbreak clusters.

The 3 independent outbreak clusters (genogroups) were only found in heterosexual individuals. Because these genogroups were not identified in samples of MSM, including bisexual men, our results make the introduction of NG in the young heterosexual population by bridging from other sexual networks unlikely, based on our data. However, 3 smaller clusters of 6 individuals were seen, and 2 contained MSM, of which 1 contained bisexual men. Bridging and mixed sexual risk groups have been described previously in strain clusters. ${ }^{9,13}$ Some strains in each genogroup of this study were also found in a study by Choudhury et al. ${ }^{9}$ and were also associated with heterosexuals likely confirming the observed clusters in this study. Choudhury et al. described significant differences in behavior and demographics of individuals between strain clusters that could indicate localized transmission clusters. In this study, the number of sex partners reported by men and CT coinfection was significantly different between the clusters. Geographical mapping in this study did not reveal clear localized clusters possibly because of low absolute numbers or relatively small geographic area (Fig. 4). In contrast, Risley et al. ${ }^{10}$ did observe strain clusters to be associated with a geographical area and considered these as localized outbreaks and persisted shorter compared with strains that were not associated with a geographical area. In this study, only one strain (G13113) was observed in 2016 (considered preoutbreak), whereas the other genogroups (G2 and GNewST) were not observed in 2016 (Fig. 3). Therefore, it is possible that the 3 clusters represent localized outbreaks in separate sexual networks but with similar epidemiological characteristics. For instance, the network could have an origin in dating app usage or attending the same school rather than location of residence.

Culture-independent molecular typing was essential for epidemiological analyses, as in total 63 of 155 samples had a cultured isolate available. The outbreak clusters might not have been identified without this method, as only 5 of 18 (G2), 2 of 11 (G13113), and 6 of 24 (GNewST) cases had a cultured isolate available for typical culture-dependent genotyping. Aside from the low sensitivity of NG culture, nucleic acid amplification tests, which use nonculturable samples, are generally used for NG detection in routine diagnostics and are not always confirmed by culture. ${ }^{3,6}$ This could lead to underestimation of linked individuals and delayed identification of outbreaks. Culture-independent molecular typing on residual nucleic acid amplification test samples could provide links between individuals rapidly to inform STI clinics.

Although 3 independent outbreak clusters were identified, targeted control measures were difficult to setup because partner information from regular partner notification to identify transmission networks of the clusters was limited. Prospective genotyping in combination with intensive contact tracing could provide the necessary data to prevent transmission in sexual networks and control NG outbreaks. However, the highly feasible and cost-effective prospective genotyping could be implemented in many places and is available for future prospective studies.

Our study does have limitations. First, the outbreak cases were analyzed and genotyped retrospectively, and therefore, we were hampered in gathering additional information. Second, only genital NG cases of clients attending the STI clinic in South-Limburg were genotyped but not clients of general practitioners, thereby potentially underestimating the magnitude of the outbreak clusters. However, groups at risk of acquiring an NG infection are mainly tested at STI-clinics. ${ }^{7}$

In conclusion, within the strong increase of NG cases in a short period, 3 independent outbreak clusters of closely related NG strains were identified using culture-free NG-MAST. This highlights the potential added value of culture-independent genotyping for outbreak investigations. Because detailed partner information was limited and we did not observe clear associations within a geographical area, additional control measures were difficult to setup. Prospective culture-independent genotyping in combination with detailed epidemiological data could provide the data needed for additional control measures to halt NG transmission and control NG outbreaks.

\section{REFERENCES}

1. Newman L, Rowley J, Vander Hoorn S, et al. Global estimates of the prevalence and incidence of four curable sexually transmitted infections in 2012 based on systematic review and global reporting. PLoS One 2015; 10:e143304.

2. Quillin SJ, Seifert HS. Neisseria gonorrhoeae host adaptation and pathogenesis. Nat Rev Microbiol 2018; 16:226-240.

3. Bignell C, Unemo M, European STI Guidelines Editorial Board. 2012 European guideline on the diagnosis and treatment of gonorrhoea in adults. Int J STD AIDS 2013; 24:85-92.

4. World Health Organisation. Global action plan to control the spread and impact of antimicrobial resistance in Neisseria gonorrhoeae. 2012.

5. World Health Organisation. WHO guidelines for the treatment of Neisseria gonorrhoeae. 2016.

6. Centers for Disease Control and Prevention. Recommendations for the laboratory-based detection of Chlamydia trachomatis and Neisseria gonorrhoeae -2014. MMWR Recomm Rep 2014; 63(RR-02):1-19.

7. Slurink IAL VAF, Heijne JCM, Op de Coul ELM, et al. Sexually Transmitted Infections in the Netherlands in 2018. Bilthoven, the Netherlands: National Institute for Public Health and Environment, 2019.

8. Town K, Bolt H, Croxford S, et al. Neisseria gonorrhoeae molecular typing for understanding sexual networks and antimicrobial resistance transmission: A systematic review. J Infect 2018; 76:507-514.

9. Choudhury B, Risley CL, Ghani AC, et al. Identification of individuals with gonorrhoea within sexual networks: A population-based study. Lancet 2006; 368:139-146.

10. Risley CL, Ward H, Choudhury B, et al. Geographical and demographic clustering of gonorrhoea in London. Sex Transm Infect 2007; 83:481-487.

11. De Silva D, Peters J, Cole K, et al. Whole-genome sequencing to determine transmission of Neisseria gonorrhoeae: An observational study. Lancet Infect Dis 2016; 16:1295-1303.

12. Fifer H, Cole M, Hughes G, et al. Sustained transmission of high-level azithromycin-resistant Neisseria gonorrhoeae in England: An observational study. Lancet Infect Dis 2018; 18:573-581.

13. Smolarchuk C, Wensley A, Padfield S, et al. Persistence of an outbreak of gonorrhoea with high-level resistance to azithromycin in England, November 2014-May 2018. Euro Surveill 2018; 23:1800287.

14. Didelot X, Dordel J, Whittles LK, et al. Genomic analysis and comparison of two gonorrhea outbreaks. MBio 2016; 7:e00525-e00516.

15. Chisholm SA, Wilson J, Alexander S, et al. An outbreak of high-level azithromycin resistant Neisseria gonorrhoeae in England. Sex Transm Infect 2016; 92:365-367.

16. Cole MJ, Thomas DR, Chisholm SA, et al. Molecular epidemiology of gonorrhoea in Wales (UK). Sex Transm Infect 2013; 89:267-272.

17. National Institute for Public Health and the Environment. Draaiboek Consult seksuele gezondheid. 2018.

18. van der Veer BM, Wolffs PF, Hoebe CJ, et al. Culture-free genotyping of Neisseria gonorrhoeae revealed distinct strains at different anatomical sites in a quarter of patients, the Netherlands, 2012 to 2016. Euro Surveill 2018; 23:1800253.

19. Chisholm SA, Unemo M, Quaye N, et al. Molecular epidemiological typing within the European Gonococcal Antimicrobial Resistance Surveillance Programme reveals predominance of a multidrug-resistant clone. Euro Surveill 2013; 18:20358.

20. Bilek N, Martin IM, Bell G, et al. Concordance between Neisseria gonorrhoeae genotypes recovered from known sexual contacts. J Clin Microbiol 2007; 45:3564-3567.

21. QGIS Development Team. 2019 QGIS Geographic Information System [computer program]. Open Source Geospatial Foundation Project.

22. Jung I, Kulldorff M, Richard OJ. A spatial scan statistic for multinomial data. Stat Med 2010; 29:1910-1918. 